\section{Safety Assessment of Endovascular Treatment of Cerebral Aneurysms in Patients with Fibromuscular Dysplasia}

\author{
Matthew T. Bender ${ }^{a} \quad$ Christopher Hurtado ${ }^{a}$ Bowen Jiang ${ }^{a}$ \\ Jessica K. Campos ${ }^{a}$ Judy Huang ${ }^{a}$ Rafael J. Tamargo ${ }^{a}$ Li-Mei Lin ${ }^{b}$ \\ Alexander L. Coon ${ }^{a}$ Geoffrey P. Colbyc \\ a Department of Neurosurgery, Johns Hopkins University School of Medicine, Baltimore, MD, \\ ${ }^{b}$ Department of Neurosurgery, University of California Irvine, Orange, CA, and ' ${ }^{\mathrm{C}}$ Department \\ of Neurosurgery, University of California Los Angeles, Los Angeles, CA, USA
}

\author{
Keywords \\ Cerebral aneurysm - Fibromuscular dysplasia - Dissection - Subarachnoid hemorrhage · \\ Flow diversion
}

\begin{abstract}
Background: The prevalence of cerebral aneurysms is increased in fibromuscular dysplasia (FMD). The presence of FMD may serve as discouragement to elective endovascular aneurysm treatment. Outcomes of endovascular intervention for aneurysms through vessels affected by FMD have not been reported. Methods: A prospectively maintained database of patients undergoing intracranial embolization was reviewed for patients with FMD who underwent endovascular aneurysm treatment. Results: A total of 1,025 patients were screened and 31 (3.0\%) had cerebrovascular FMD. These patients underwent a total of 43 embolization procedures; 27 of these procedures were performed through an affected vessel. All but 1 patient were female and the average age was 62 years. "String-of-pearls"-type FMD was the most common subtype (90\%). The internal carotid arteries were more commonly affected (65\%) than the vertebral arteries (48\%). All patients underwent treatment of cerebral aneurysms, most of which (87\%) were incidentally discovered; 6 patients (19\%) also had incidental vessel dissection. The average aneurysm size was $7.1 \mathrm{~mm}$. The morphology was saccular in $93 \%$ of the cases, and $86 \%$ were in the anterior circulation. The most commonly performed treatment was flow diversion (67\%), in the majority of cases by pipeline embolization. Other procedures performed were coiling (19\%), stent-coiling (12\%), and intrasaccular flow disruption (2\%). All but 1 procedure (98\%) were successful. There were no major complications; 1 patient experi-
\end{abstract}


enced a transient ischemic attack. Follow-up angiography was performed in $88 \%$ of the cases, without evidence for disease progression after treatment. The average time to last angiographic follow-up was 17 months $( \pm 13)$. Conclusions: Elective embolization of intracranial aneurysms can be performed safely through vessels affected by FMD. @ 2017 S. Karger AG, Basel

\section{Introduction}

Fibromuscular dysplasia (FMD) is an arteriopathy affecting multiple vascular beds throughout the body and commonly the mid-cervical carotid and vertebral arteries. The medial type, the most common histopathology, is characterized on angiography by alternating fibrous constrictions and aneurysmal dilations, commonly referred to as "string of beads" [1]. Of relevance to neurosurgeons and neurointerventionalists, the prevalence of cervical dissection and intracranial aneurysms is increased in patients with FMD [2-4]. As more such lesions are incidentally discovered, the presence of FMD can serve as discouragement to elective treatment of cerebral aneurysms [5]. There are no reports of outcomes of endovascular interventions through vessels affected by FMD.

For this study, we reviewed a prospectively maintained database of patients undergoing intracranial embolization to identify patients with FMD who underwent treatment, and assessed disease severity, procedural outcomes, and progression after treatment.

\section{Methods}

The study included a retrospective cohort of patients utilizing an institutional review board-approved, prospectively collected database of patients undergoing endovascular embolization procedures at a tertiary medical center. Four- or six-vessel diagnostic cerebral angiogram reports by experienced neuroradiologists were reviewed to identify patients with FMD, and the diagnosis was subsequently confirmed by review of digital subtraction angiography images. Demographic information, clinical history data, and outcomes were collected from medical records. Anatomic and technical details were collected from intraprocedural events, angiograms, and operative reports. The angiographic appearance of FMD was classified according to the system originally described by Osborn and Anderson [1]. Embolization procedures were performed as previously described [6-8] through femoral access with a triaxial catheter system including a guide sheath, distal intracranial catheter, and microcatheter. Patients recovered in the neurocritical care unit and were typically discharged home on postembolization day 1. Data are presented as means and range for continuous variables and as frequency for categorical variables.

\section{Results}

Cerebral angiogram reports were screened for a total of 1,025 patients who underwent embolization procedures, and 31 patients (3.0\%) were identified and subsequently confirmed as having cerebrovascular FMD. These patients underwent a total of 43 embolization procedures. Among the patients having more than one procedure, 6 had multiple distinct aneurysms treated, 2 patients underwent staged stent-coiling procedures, 2 underwent flow diversion for residual aneurysm neck filling after coiling during the acute stage of subarachnoid hemorrhage (SAH), and 2 underwent a second flow diversion procedure for persistent aneurysm filling. A total of 19 patients had treatment through an affected vessel, and 27 procedures were performed through an affected vessel. All but 1 patient were female, a majority were white $(77 \%)$, and the average age was 62 years. "String-of-pearls"-type FMD 
Table 1. Demographics and FMD characteristics

\begin{tabular}{|c|c|c|}
\hline & $n$ & $\%$ \\
\hline FMD patients & 31 & 3.0 \\
\hline FMD cases & 43 & \\
\hline Mean age \pm SD (range), years & $61.7 \pm 11.4(46-89)$ & \\
\hline Female gender & 30 & 96.8 \\
\hline \multicolumn{3}{|l|}{ Race } \\
\hline White & 24 & 77.4 \\
\hline Black & 6 & 19.4 \\
\hline Hispanic & 1 & 3.2 \\
\hline \multicolumn{3}{|l|}{ FMD type } \\
\hline String of beads & 28 & 90.3 \\
\hline Tubular stenosis & 1 & 3.2 \\
\hline Atypical & 2 & 6.5 \\
\hline \multicolumn{3}{|l|}{ Vessels affected } \\
\hline 1 & 1 & 3.2 \\
\hline 2 & 15 & 48.4 \\
\hline 3 & 9 & 29.0 \\
\hline 4 & 6 & 19.4 \\
\hline \multicolumn{3}{|l|}{ Associated pathology } \\
\hline Cerebral aneurysm & 31 & 100.0 \\
\hline Dissection & 6 & 19.4 \\
\hline \multicolumn{3}{|l|}{ Presentation } \\
\hline Subarachnoid hemorrhage & 4 & 12.9 \\
\hline Stroke & 0 & 0.0 \\
\hline Incidental & 27 & 87.1 \\
\hline
\end{tabular}

was the most common subtype (90\%), while tubular stenosis (3\%) and atypical FMD (7\%) were found in a minority of patients. All but 1 patient had more than one vessel affected. All patients underwent treatment of cerebral aneurysms, most of which (87\%) were incidentally discovered; 6 patients (19\%) also had incidental vessel dissection (Table 1).

The internal carotid arteries were more commonly affected $(65 \%)$ than the vertebral arteries (48\%), with overlapping involvement of the anterior and posterior circulation in $17 \%$ of the cases. The cervical segment was involved in most patients with internal carotid artery (ICA) involvement. The petrous segment was involved in less than $10 \%$ of the patients, and cavernous or intracranial ICA FMD was not seen. The most commonly affected segment of the vertebral artery was V3 (44\%), followed by V2 (24\%). The V4 segment was involved in a minority of patients (10\%), and intracranial involvement was not seen. In each case, the average length of the vessel affected was between 2.5 and $3.2 \mathrm{~cm}$ (Table 2).

The average size of a treated aneurysm was $7.1 \pm 3.6 \mathrm{~mm}$. The morphology was saccular in $40 / 42$ cases (93\%). Aneurysms were located in the anterior circulation in 37 cases (86\%), and along the ICA in 33 cases and the anterior cerebral artery in 4 cases. The most common anterior circulation aneurysm locations were true ophthalmic $(n=9)$, paraophthalmic $(n=$ 9 ), in the posterior communicating artery $(n=4)$, in the supraclinoid ICA $(n=4)$, and in the anterior communicating artery $(n=3)$. Aneurysms were located in the posterior circulation in 6 cases $(86 \%)$, 4 of which were basilar apical aneurysms.

The most commonly performed treatment was flow diversion, which was performed in 29 cases (67\%), in the majority of cases by pipeline embolization. Other procedures performed were coiling (19\%), stent-coiling (12\%), and intrasaccular flow disruption (2\%). The average fluoroscopy time was $40 \mathrm{~min}$ and the radiation dose was 2,095 mGy. All but 1 procedure (98\%) were successful. Verapamil was administered for vasospasm in 3 cases (7\%), balloon 
Table 2. Vessel involvement

\begin{tabular}{|c|c|c|c|c|}
\hline & \multicolumn{2}{|c|}{ Right } & \multicolumn{2}{|c|}{ Left } \\
\hline & $n$ & $\%$ & $n$ & $\%$ \\
\hline Any ICA involvement & 21 & 67.7 & 19 & 61.3 \\
\hline Cervical & 21 & 67.7 & 18 & 58.1 \\
\hline Petrous & 2 & 6.5 & 3 & 9.7 \\
\hline Cavernous/intracranial & 0 & 0.0 & 0 & 0.0 \\
\hline \multicolumn{5}{|c|}{ Average ICA length involved \pm SD } \\
\hline (range), $\mathrm{cm}$ & \multicolumn{2}{|c|}{$3.0 \pm 1.8(1.3-7.5)$} & \multicolumn{2}{|c|}{$3.2 \pm 2.0(1.0-9.5)$} \\
\hline Any vertebral involvement & 15 & 48.4 & 15 & 48.4 \\
\hline V2 & 9 & 29.0 & 6 & 19.4 \\
\hline V3 & 13 & 41.9 & 14 & 45.2 \\
\hline V4 & 4 & 12.9 & 2 & 6.5 \\
\hline Intracranial & 0 & 0.0 & 0 & 0.0 \\
\hline \multicolumn{5}{|l|}{ Average vertebral length \pm SD } \\
\hline (range), $\mathrm{cm}$ & \multicolumn{2}{|c|}{$2.8 \pm 1.6(1.3-6.5)$} & \multicolumn{2}{|c|}{$2.5 \pm 1.2(0.7-4.5)$} \\
\hline
\end{tabular}

ICA, internal carotid artery.

Table 3. Procedural details and outcomes

Procedure type

Flow diversion

Coiling

Stent-coiling

Intrasaccular flow disruption

Mean fluoroscopy time \pm SD (range), min

Mean radiation dose \pm SD (range), $m G y$

Success

Spasm/verapamil

Balloon angioplasty

Rupture/perforation

Thrombosis

Complications

Transient ischemic attack

Stroke

Intracranial hemorrhage

Subarachnoid hemorrhage

Dissection

Groin hematoma

Death n $\%$

$\begin{array}{rr}29 & 67.4 \\ 8 & 18.6 \\ 5 & 11.6 \\ 1 & 2.3 \\ 40.3 \pm 19(17-88) & \\ 2,095 \pm 936(824-5,113) & 97.7 \\ 42 & 7.0 \\ 3 & 11.6 \\ 5 & 0.0 \\ 0 & 2.3 \\ 1 & \\ & \\ 1 & 2.3 \\ 0 & 0.0 \\ 0 & 0.0 \\ 0 & 0.0 \\ 0 & 0.0 \\ 1 & 2.3 \\ 0 & 0.0\end{array}$

angioplasty was required in 5 cases (12\%), and stent thrombosis, which resolved with abciximab administration, was seen in 1 case (2\%). There were no major complications such as stroke, $\mathrm{SAH}$, intracranial hemorrhage, and death. No iatrogenic dissections occurred. Minor complications included 1 patient with a transient ischemic attack without any imaging correlate, and 1 patient with groin hematoma that did not require transfusion (Table 3). Follow-up angiography was performed in $88 \%$ of the cases, without evidence for FMD disease progression after treatment. Complete aneurysm occlusion with no contrast opacification 
was observed in $22 / 28$ patients (79\%) who underwent flow diversion with pipeline embolization. The average time to last angiographic follow-up was 17 months $( \pm 13)$.

\section{Discussion}

In this retrospective study of more than 1,000 patients undergoing intracranial embolization procedures, predominantly for aneurysm, the prevalence of FMD was 3.0\%. The patients with FMD underwent a total of 43 embolization procedures, 27 of which were performed on 19 patients through a vessel affected by FMD. Clinically, there were no major complications. Angiographically, follow-up imaging was performed on $88 \%$ of the patients, with no evidence of disease progression. Our results demonstrate the safety of elective endovascular treatment of intracranial aneurysms in patients with FMD.

Patients with FMD have increased rates of arterial dissection and cerebral aneurysms. Historically, FMD was commonly diagnosed in patients undergoing cerebral angiography for $\mathrm{SAH}$, leading to inflated estimates of the aneurysm prevalence in FMD on the order of $20-50 \%$ $[9,10]$. In a meta-analysis, Cloft et al. [2] removed patients with SAH from FMD series and found the prevalence of asymptomatic unruptured intracranial aneurysms to be $7.3 \%$. The reported prevalence of unruptured intracranial aneurysm in the US registry for FMD was $4.6 \%$, although only $64 \%$ of the patients had any intracranial vascular imaging and there were patients not included in this number who reported a history of SAH without a known aneurysm. By including the extracranial carotid and vertebral artery, the prevalence of aneurysms rose to $12.3 \%$ and the prevalence of dissection along the carotid or vertebral artery was $21.7 \%$ [3]. A more recent report from the US FMD registry found that $12.9 \%$ of women with FMD who had vascular imaging had an intracranial aneurysm [4]. Even after adjusting for the preponderance of women among people with FMD and people with aneurysm, the prevalence of aneurysms among FMD patients exceeds the population average, which is between 0.4 and $6.0 \%$, higher in prospective angiographic studies and lower in retrospective autopsy studies [11]. Given the increased prevalence of aneurysms among patients with FMD, multiplicity is not a surprising finding, and indeed 6 patients in this series underwent separate treatments of distinct aneurysms.

Cerebral aneurysms in patients with FMD are a potentially unique pathology whose natural history warrants study to determine risks and benefits of elective treatment. True saccular aneurysms lack a tunica media and internal elastic membrane, whereas vessels affected by FMD - including the circle of Willis, though it is rarely apparent on angiography show disordered fibroplasia within the tunica media [12]. These processes could compound or neutralize the rupture risk of intracranial aneurysms; yet their natural history in patients with FMD has not been independently characterized. Cloft et al. [2] sought to calculate the prevalence of aneurysms in FMD by removing patients with SAH from the equation. This assumes FMD aneurysms do not pose a different risk of rupture, an assumption the authors acknowledged and thought reasonable in the absence of evidence to the contrary. Study leaders from the US FMD registry more openly acknowledged the need to use existing guidelines regarding when to treat unruptured aneurysms in the absence of specific data on aneurysms in patients with FMD [3]. At least one study has suggested that once an aneurysm ruptures in a patient with FMD, there may be higher rates of vasospasm that lead to worsened outcomes [13].

There would seem to be a heightened risk of iatrogenic injury associated with endovascular treatment through fragile and tortuous FMD vessels, but reports are limited. Fuse et al. [5] shared the case of a 30-year-old male with SAH from a ruptured posterior inferior cerebellar artery aneurysm who underwent vessel sacrifice and multiple transcatheter vaso- 
Fig. 1. Digital subtraction angiograms. a Anterior-posterior view of the proximal internal carotid artery hairpin loop and string-ofbeads fibromuscular dysplasia (FMD) affecting the cervical internal carotid artery (ICA). b Midembolization lateral roadmap with a guide sheath proximal to the FMD within the distal common carotid and a distal access catheter (black arrowhead) within the mid-horizontal segment of a type IV cavernous ICA. c Preembolization lateral angiogram showing an 8-mm supraclinoid ICA aneurysm. d Pipeline embolization deployment across the neck of the aneurysm. e Sixmonth follow-up lateral angiogram showing complete aneurysm occlusion without evidence of FMD progression.

Bender et al.: Safety Assessment of Endovascular Treatment of Cerebral Aneurysms in Patients with Fibromuscular Dysplasia

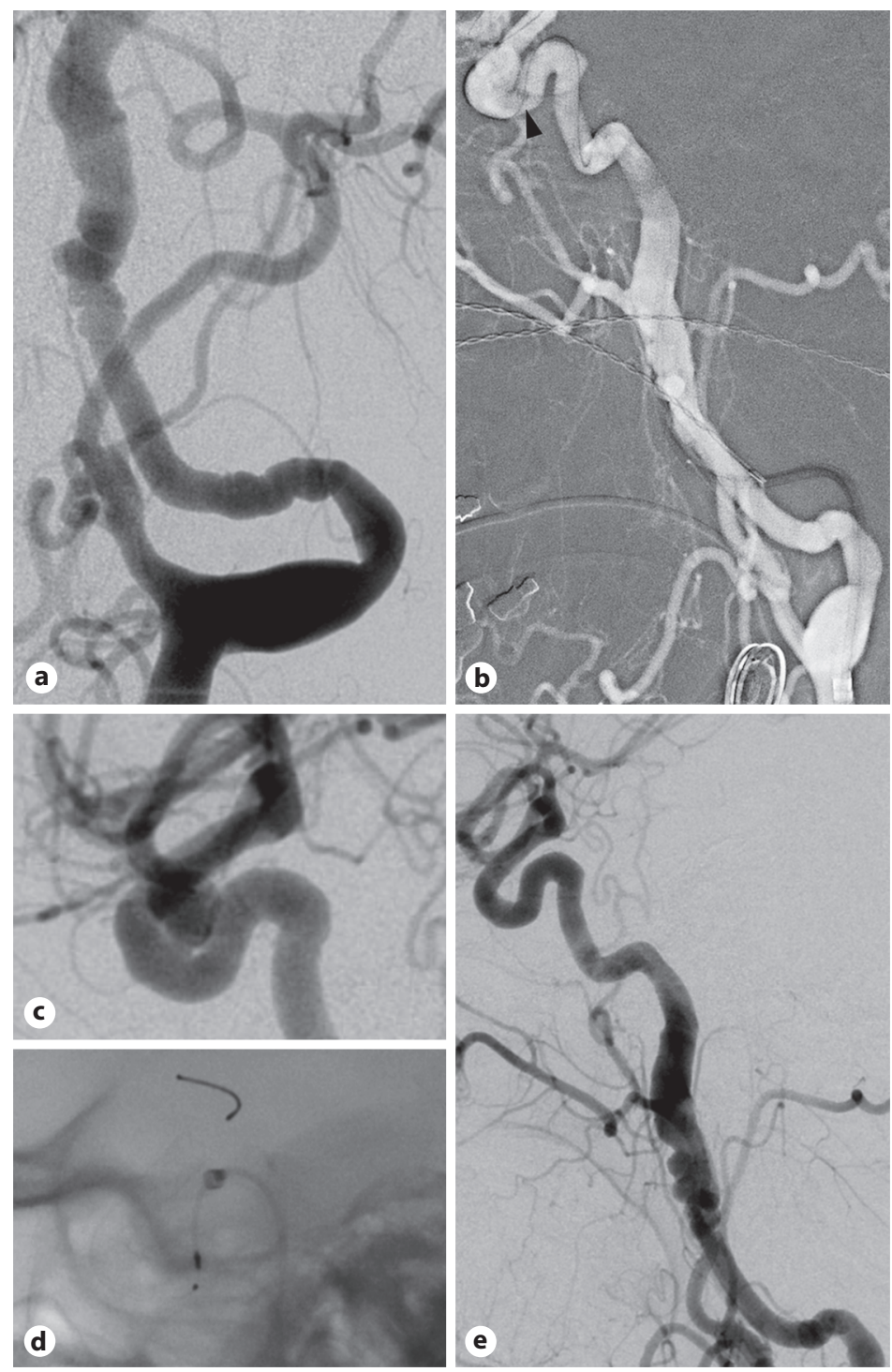

spasm treatments, and who then developed a 3-cm iatrogenic dissecting aneurysm of the external carotid artery. The authors concluded that particular care is needed when performing endovascular interventions in the presence of FMD. An unacknowledged reluctance to treat through FMD may be evidenced in the relative minority of intracranial aneurysms in the US FMD registry to undergo elective treatment (36\%) and the abiding reliance on surgical clipping (43\%) [3], both of which counter national trends toward elective endovascular treatment of unruptured aneurysms [14]. That registry reports elective treatment of 16 intracranial aneurysms in patients with FMD, but it does not break down open versus elective treatment, procedural outcomes, and whether the aneurysm was located on a vessel with angiographically apparent FMD [3]. Similarly, a Belgian series of 123 patients with FMD likewise reports coiling of 7 incidentally discovered cerebral aneurysms without reporting outcomes [15]. Our series presents the first reported outcomes of treating dependent aneurysms through FMD-affected vessels. We performed 43 embolization procedures on patients with FMD, 27 through a vessel showing angiographic signs of disease without major complications or signs of progression on follow-up imaging. 
To achieve these outcomes, no matter the aneurysm location or endovascular treatment modality, catheter access is a universally important technical consideration in treating patients with FMD. We routinely use a triaxial system for intracranial embolization, with minimal modification for FMD cases, as in the illustrative case of a quinquagenarian with an 8-mm left-sided supraclinoid ICA aneurysm treated with the pipeline embolization device (Fig. 1). For anterior circulation procedures, a 6-Fr guide sheath such as the AXS Infinity or NeuronMax is advanced to the carotid bifurcation, which is typically spared of FMD features [16]. Distal positioning of the guide sheath is essential to support the navigation of contemporary flexible distal intracranial catheters through the alternating fibrous bands and dilations of FMD as well as the common loops in the cervical ICA. Limiting step-off between coaxial catheters helps reduce the risk of dissection. The diseased segment is navigated under high magnification with a device-specific microcatheter and often a J-tipped $0.014^{\prime \prime}$ microwire. The hydrophilic coating and rounded tips of contemporary distal intracranial catheters such as the Catalyst 5 [17] minimize vessel wall trauma while the catheter is tracked beyond the diseased segment to the cavernous ICA at a minimum. Prophylactic use of an intra-arterial spasmolytic such as verapamil can also be helpful to limit vasospasm in and around FMD segments. When possible, device deployment is performed under demagnified views, allowing constant observation for catheter construct stability in the cervical segment.

The patients included in this study constitute a representative sample of patients with FMD. They were predominantly middle-aged, female, and white $[18,19]$. Most patients had more than one vessel affected. Cervical ICA involvement was the most common, although vertebral involvement was more common than in most series, which is estimated at $10-36 \%$ $[1,19]$. This may be due to the improved resolution achieved by digital subtraction angiography, which was used for all patients in this series, compared to ultrasound, MRA, and CTA, which were used in a majority of cases in other studies $[19,20]$, on which bony landmarks obscure the assessment of the vertebral artery, especially around the V3 segment. Although multiple angiographic subtypes of FMD can be observed in a single patient, the string-ofbeads appearance predominated in more than $90 \%$ of the cases in this series, as is typical in the literature [19].

Although the focus of this study is on the safety of a variety of endovascular embolization procedures in the setting of FMD, there is reason to think that FMD patients may differ from the general population in healing and regrowth after aneurysm embolization. The most commonly performed procedure reported in this series was flow diversion with the pipeline embolization device (29 cases). Aneurysm obliteration was achieved in 22/28 patients (79\%), with angiographic follow-up at a mean interval of 17 months. This is similar to our observation of complete occlusion in $78 \%$ of all patients undergoing anterior circulation pipeline embolization for cerebral aneurysm at 12-month follow-up angiography (forthcoming data).

Limitations of this study include defining FMD angiographically without any corresponding histopathology [19]. It has long been accepted that the most commonly observed angiographic subtype (string of beads) corresponds to the most commonly observed histopathological subtype (medial) [1]. The string-of-beads phenotype is relatively easily distinguished from stationary waves, vasospasm, or atherosclerosis by the presence of dilations larger than the normal parent vessel and by its relative isolation to the cervical region; however, the less common phenotypes - tubular stenosis and atypical FMD - have a less specific radiographic appearance, which may lead to their underdiagnosis in this and other series. Additionally, due to our status as a referral center, angiographic follow-up was not complete but was obtained for only $88 \%$ of the patients. 


\section{Conclusions}

Cerebral aneurysms are common among patients with FMD. Endovascular treatment of aneurysms through vessels affected by FMD is technically challenging, but it can be done safely.

\section{Disclosure Statement}

A.L. Coon is a consultant and proctor for Medtronic, Stryker, and MicroVention. G.P. Colby is a consultant for MicroVention and Codman.

\section{References}

1 Osborn AG, Anderson RE: Angiographic spectrum of cervical and intracranial fibromuscular dysplasia. Stroke 1977;8:617-626.

2 Cloft HJ, Kallmes DF, Kallmes MH, Goldstein JH, Jensen ME, Dion JE: Prevalence of cerebral aneurysms in patients with fibromuscular dysplasia: a reassessment. J Neurosurg 1998;88:436-440.

3 Kadian-Dodov D, Gornik HL, Gu X, Froehlich J, Bacharach JM, Chi Y-W, et al: Dissection and aneurysm in patients with fibromuscular dysplasia: findings from the US Registry for FMD. J Am Coll Cardiol 2016;68: 176-185.

4 Lather HD, Gornik HL, Olin JW, Gu X, Heidt ST, Kim ESH, et al: Prevalence of intracranial aneurysm in women with fibromuscular dysplasia: a report from the US Registry for Fibromuscular Dysplasia. JAMA Neurol 2017; 74:1081-1087.

5 Fuse T, Umezu M, Yamamoto M, Demura K, Nishikawa Y, Niwa Y: External carotid artery aneurysm developing after embolization of a ruptured posterior inferior cerebellar artery aneurysm in a patient with cervicocephalic fibromuscular dysplasia - case report. Neurol Med Chir (Tokyo) 2006;46:290-293.

6 Colby GP, Lin L-M, Gomez JF, Paul AR, Huang J, Tamargo RJ, et al: Immediate procedural outcomes in 35 consecutive pipeline embolization cases: a single-center, single-user experience. J Neurointerv Surg 2013;5:237246.

7 Colby GP, Lin L-M, Caplan JM, Jiang B, Huang J, Tamargo RJ, et al: Immediate procedural outcomes in 44 consecutive Pipeline Flex cases: the first North American single-center series. J Neurointerv Surg 2016;8:702-709.

8 Bender MT, Lin L-M, Colby GP, Lubelski D, Huang J, Tamargo RJ, et al: P2Y12 hyporesponse (PRU >200) is not associated with increased thromboembolic complications in anterior circulation Pipeline. J Neurointerv Surg 2017;9:978-981.

9 Mettinger KL: Fibromuscular dysplasia and the brain. II. Current concept of the disease. Stroke 1982;13: 53-58.

10 Touzé E, Oppenheim C, Trystram D, Nokam G, Pasquini M, Alamowitch S, et al: Fibromuscular dysplasia of cervical and intracranial arteries. Int J Stroke 2010;5:296-305.

11 Rinkel GJ, Djibuti M, Algra A, van Gijn J: Prevalence and risk of rupture of intracranial aneurysms: a systematic review. Stroke 1998;29:251-256.

12 Lummus S, Breeze R, Lucia MS, Kleinschmidt-DeMasters BK: Histopathologic features of intracranial vascular involvement in fibromuscular dysplasia, Ehlers-Danlos type IV, and neurofibromatosis I. J Neuropathol Exp Neurol 2014;73:916-932.

13 George B, Zerah M, Mourier KL, Gelbert F, Reizine D: Ruptured intracranial aneurysms. The influence of sex and fibromuscular dysplasia upon prognosis. Acta Neurochir (Wien) 1989;97:26-30.

14 Smith GA, Dagostino P, Maltenfort MG, Dumont AS, Ratliff JK: Geographic variation and regional trends in adoption of endovascular techniques for cerebral aneurysms. J Neurosurg 2011;114:1768-1777.

15 De Groote M, Van der Niepen P, Hemelsoet D, Callewaert B, Vermassen F, Billiouw J-M, et al: Fibromuscular dysplasia - results of a multicentre study in Flanders. Vasa 2017;46:211-218.

16 Lin L-M, Bender MT, Colby GP, Beaty NB, Jiang B, Campos JK, et al: Use of a next-generation multi-durometer long guide sheath for triaxial access in flow diversion: experience in 95 consecutive cases. J Neurointerv Surg 2017, Epub ahead of print.

17 Colby GP, Lin L-M, Xu R, Beaty N, Bender MT, Jiang B, et al: Utilization of a novel, multi-durometer intracranial distal access catheter: nuances and experience in 110 consecutive cases of aneurysm flow diversion. Interv Neurol 2017;6:90-104.

18 Lemahieu SF, Marchau MM: Intracranial fibromuscular dysplasia and stroke in children. Neuroradiology 1979;18:99-102.

19 Olin JW, Froehlich J, Gu X, Bacharach JM, Eagle K, Gray BH, et al: The United States Registry for Fibromuscular Dysplasia: results in the first 447 patients. Circulation 2012;125:3182-3190.

20 Olin JW, Sealove BA: Diagnosis, management, and future developments of fibromuscular dysplasia. J Vasc Surg 2011;53:826-836.e1. 